\title{
Examining the Physical Conditions of a Warm Corona in Active Galactic Nuclei Accretion Discs
}

\author{
D. R. Ballantyne* \\ Center for Relativistic Astrophysics, School of Physics, Georgia Institute of Technology, 837 State Street, Atlanta, GA 30332-0430, USA
}

Accepted XXX. Received YYY; in original form ZZZ

\begin{abstract}
A warm corona at the surface of an accretion disc has been proposed as a potential location for producing the soft excess commonly observed in the X-ray spectra of active galactic nuclei (AGNs). In order to fit the observed data the gas must be at temperatures of $\sim 1 \mathrm{keV}$ and have an optical depth of $\tau_{\mathrm{T}} \approx 10-20$. We present one-dimensional calculations of the physical conditions and emitted spectra of a $\tau_{\mathrm{T}}=10$ or 20 gas layer subject to illumination from an $\mathrm{X}$-ray power-law (from above), a blackbody (from below) and a variable amount of internal heating. The models show that a warm corona with $k T \sim 1 \mathrm{keV}$ can develop, producing a strong Comptonized soft excess, but only if the internal heating flux is within a relatively narrow range. Similarly, if the gas density of the layer is too large then efficient cooling will stop a warm corona from forming. The radiation from the hard X-ray power-law is crucial in producing a warm corona, indicating that a warm and hot corona may co-exist in AGN accretion discs, and their combined effect leads to the observed soft excess. Intense heating of a warm corona leads to steep X-ray spectra with ionised $\mathrm{Fe} \mathrm{K} \alpha$ lines, similar to those seen in some narrow-line Seyfert 1 galaxies.
\end{abstract}

Key words: galaxies: active - X-rays: galaxies - accretion, accretion discs - galaxies: Seyfert

\section{INTRODUCTION}

The X-ray spectra of active galactic nuclei (AGNs) at energies $\gtrsim 2 \mathrm{keV}$ are often well described by a cutoff power-law continuum (e.g. Panessa et al. 2008; Malizia et al. 2014; Ricci et al. 2017, 2018; Molina et al. 2019) that is consistent with being produced by Comptonization of ultraviolet (UV) disc photons in a hot, tenuous corona (e.g., Galeev et al. 1979; Haardt \& Maraschi 1991, 1993; Jiang et al. 2014, 2019b). However, below this energy the majority of AGNs ( $\gtrsim 50 \%$; e.g., Scott et al. 2012; Winter et al. 2012; Ricci et al. 2017) exhibit a significant amount of emission above what is predicted by extrapolating the power-law to lower-energies. This 'soft excess' has been observed in many AGNs for decades (e.g., Arnaud et al. 1985; Pounds et al. 1986; Walter \& Fink 1993; Leighly 1999), but the origin of this emission is not understood. Observationally, the soft excess appears as a featureless, smoothly varying continuum that can be described by thermal emission with temperatures $\sim 0.1-0.2 \mathrm{keV}$ (e.g., Bianchi et al. 2009). The blackbody temperatures do not show any correlation with the AGN black hole masses or accretion rates, indicating that the emission is not simply thermal radiation from the hottest part of the accretion disc (e.g., Gierliński \& Done 2004; Bianchi et al. 2009).

The lack of variation observed in the temperatures has led to models where the soft excess is strongly influenced by atomic

^ E-mail: david.ballantyne@physics.gatech.edu processes (e.g., Gierliński \& Done 2004). For example, reprocessing of the X-ray power-law in the inner accretion disc (i.e., Xray 'reflection'; Fabian \& Ross 2010) naturally predicts a strong bremsstrahlung spectrum at energies $\lesssim 1 \mathrm{keV}$ that may be accompanied by a forest of spectral lines depending on the ionization state of the reflecting region (e.g., Ross \& Fabian 1993; Ballantyne et al. 2001b; García \& Kallman 2010). If this reflection spectrum is produced close to a rapidly spinning black hole, light-bending effects will combine with strong relativistic blurring of the spectrum to produce a featureless soft excess that can describe the observations in some cases (e.g., Crummy et al. 2006; Walton et al. 2013; García et al. 2019). However, the significant relativistic blurring needed by the model to explain the featureless soft excess often leads to solutions with extremely rapidly spinning black holes and very low coronal heights, even with models that allow for large disc densities (e.g., García et al. 2019; Jiang et al. 2019a). While it is clear that soft emission from the disc reflection spectrum must contribute to the soft excess in many AGNs, the fact that models are pushed into an extreme corner of parameter space is concerning. Spectral modeling that allows for an extra emission component to help with the soft excess fit does not require such extreme values for the black hole and corona properties (e.g., Keek \& Ballantyne 2016). Therefore, it is worth considering how this extra emission component will interact with the disc reflection physics.

An alternative model for the soft excess returns to the idea of direct emission from the AGN accretion disc, but now proposes 
that the thermal disc emission passes through a thick $\left(\tau_{\mathrm{T}} \sim 10\right.$ 20 , where $\tau_{\mathrm{T}}$ is the optical depth to Thomson scattering), warm ( $k T \sim 0.1-1 \mathrm{keV}$ ) Comptonizing layer (e.g., Magdziarz et al. 1998; Czerny et al. 2003; Petrucci et al. 2013, 2018; Kubota \& Done 2018). If this layer is common in AGNs, then the thermal disc emission will be broadened up to soft X-ray energies, and, depending on how the warm layer is heated, may appear insensitive to changes in the black hole mass and accretion rate. This 'warm corona' would need to reside at or near the surface of the accretion disc, and could be heated by accretion energy dissipated within the disc and transported vertically (perhaps by magnetic fields; e.g., Jiang et al. 2019b) to the disc surface. Petrucci et al. (2018) used a phenomenological warm corona model to successfully fit nearly 100 optical-to-X-ray spectra of 22 Seyfert galaxies observed with $X M M-N e w t o n$. However, it is unclear if this scenario is physically plausible. For example, Różańska et al. (2015) considered the hydrostatic balance of a warm corona on top of a cool accretion disc and found that balance was only achieved for $\tau_{\mathrm{T}}<5$ without additional pressure support from, e.g., magnetic fields. More recently, García et al. (2019) showed that gas at the temperatures and optical depths expected in a warm corona will retain significant photoelectric opacity and therefore any radiation passing through the gas will be imprinted with many soft X-ray absorption lines which are not observed in AGN data.

Despite these difficulties, the substantial uncertainty in our understanding in how accretion energy is transported and dissipated through a disc-corona system means that it is important to continue to closely examine the warm corona scenario. In this paper, the atomic and radiative processes within a warm corona are treated in detail in order to accurately predict its radiative signatures, and compare them against those needed in the phenomenological models (e.g., Petrucci et al. 2018). In particular, we note that the warm corona scenario still requires the presence of the hard X-ray powerlaw and its associated reflection spectrum. In fact, the X-ray powerlaw will be illuminating the surface of any warm corona, significantly impacting the physical conditions within that layer. It is important, therefore, to consider the effect of the power-law heating on the physical properties of a warm corona and to determine if this situation can still provide an explanation for the soft excess in AGNs. This paper investigates this question by computing the physical conditions of a layer of gas subject to (a) uniform heating throughout by an assumed accretion flux, (b) an external hard Xray power-law, and (c) a soft blackbody injected from below. These calculations will determine under which conditions, if any, a warm corona with properties similar to those inferred from observations can exist when including the effects of the power-law irradiation. The next section describes the set-up of the calculation, with the results presented in Sect. 3 and discussed in Sect. 4. The last section (Sect. 5) summarizes the key findings and conclusions.

\section{CALCULATIONS}

The calculations are based on the one-dimensional, timeindependent reflection models of Ballantyne et al. (2001b, 2002) in which the surface of the accretion disc, treated as a slab of gas with hydrogen number density $n_{\mathrm{H}}$ and Thomson depth $\tau_{\mathrm{T}}$, is irradiated by an external X-ray power-law. A blackbody spectrum can also be introduced into the lowest zone of the slab to account for the emission from the bulk of the accretion disc laying below the slab. As described in the above articles and references therein, the temperature structure of the slab is solved by considering multiple heating and cooling processes in the gas, and the spectrum emitted by the surface (comprising the sum of the reprocessed power-law radiation, transfered blackbody flux, and diffuse emission from the gas) is computed once the slab reaches thermal balance. The temperature of AGN accretion discs is expected to be large enough that hydrogen is completely ionized (Shakura \& Sunyaev 1973) filling the slab with free electrons. Thus, the calculation carefully treats the effects of Compton scattering which is crucial in redistributing photon energies and shaping the emitted spectrum (Ross 1979; Ross \& Fabian 1993). This fact, combined with the broad energy range treated by the calculations $(0.8 \mathrm{eV}$ to $98 \mathrm{keV})$, allows for the simultaneous prediction of both the soft-excess and hard X-ray reflection properties produced by an irradiated disc surface.

In order to self-consistently predict the spectrum emitted by an optically-thick warm corona in the presence of a hard X-ray powerlaw, we consider the total emergent flux emitted by an accretion disc (per disc side) at radius $R, D(R)$. Following the warm corona hypothesis, a fraction $h_{f}$ of this flux is assumed to be uniformly dissipated as heat throughout the irradiated slab. This leads to an additional heating function (in $\mathrm{erg} \mathrm{cm}^{3} \mathrm{~s}^{-1}$ ) in the calculation,

$\mathscr{H}=\frac{h_{f} D(R) \sigma_{\mathrm{T}}}{\tau_{\mathrm{T}} n_{\mathrm{H}}}$

where $\sigma_{\mathrm{T}}$ is the Thomson cross-section. This heating function corresponds to a constant heating rate per particle over $\tau_{T}$.

The hard X-ray power-law that irradiates the disc is assigned a total flux of $f_{X} D(R)$, following the expectation that the hot corona is heated by accretion energy transported outside of the disc (e.g., Jiang et al. 2014, 2019b). In order to consider a large range of $h_{f}$, we fix $f_{X}=0.1$, a value similar to the X-ray bolometric corrections inferred in local AGNs (e.g., Vasudevan \& Fabian 2007; Netzer 2019). The illuminating hard X-ray power-law is defined to have an exponential cutoff at $30 \mathrm{eV}$ to mimic its expected origin as Comptonized accretion disc emission (e.g., Coppi 1999). In addition, both the photon-index of the power-law, $\Gamma$, and its high-energy cutoff energy, $E_{\text {cut }}$ are fixed at $\Gamma=1.9$ and $E_{\text {cut }}=220 \mathrm{keV}$, respectively. These values are typical of bright Seyfert galaxies (e.g., Ballantyne 2014; Ricci et al. 2017, 2018). Finally, the remaining flux, $\left(1-f_{X}-h_{f}\right) D(R)$, is injected into the bottom of the slab as a blackbody with a temperature given by the standard blackbody formula. This setup allows an investigation of the emitted spectrum produced by the heated layer of an accretion disc while constraining the total energy available for heating the gas.

The accretion flux $D(R)$ is calculated from the standard thin disc equation (e.g. Shakura \& Sunyaev 1973; Merloni et al. 2000) with a black hole mass of $2.5 \times 10^{7} \mathrm{M}_{\odot}$, an accretion rate of 0.6 times the Eddington value, and $R=5$ Schwarzschild radii. These values result in $D(R)=5.175 \times 10^{16} \mathrm{erg} \mathrm{cm}^{-2} \mathrm{~s}^{-1}$. Spectral fitting results using the warm corona hypothesis find that the heated layer has $\tau_{\mathrm{T}} \approx 10-20$ (e.g., Petrucci et al. 2018). In addition, the heating and cooling processes in the gas (e.g., Compton scattering, bremsstrahlung) are strong functions of density, so the spectra produced by the models will depend on the assumed density of the slab (e.g., Ballantyne 2004; García et al. 2016; Jiang et al. 2019a). Therefore, the following set of four scenarios are considered to explore the properties of a warm corona: $n_{\mathrm{H}}=2 \times 10^{14} \mathrm{~cm}^{-3}$ $\left(\tau_{\mathrm{T}}=10,20\right)$ and $n_{\mathrm{H}}=5 \times 10^{14} \mathrm{~cm}^{-3}\left(\tau_{\mathrm{T}}=10,20\right)$. Eight reflection models, from $h_{f}=0.1$ to 0.8 , are computed for each $\left(n_{\mathrm{H}}\right.$, $\tau_{\mathrm{T}}$ ) pair. The resulting X-ray emission from and physical conditions within the model warm coronae are discussed below. 


\section{RESULTS}

The goals of the calculations are to (1) test the likelihood of forming a $k T \sim 1 \mathrm{keV}$ warm corona when the heated slab is subject to irradiation from a hard X-ray power-law, and (2) determine if the resulting emission and reflection spectrum exhibits a strong, relatively smooth soft excess. The models are evaluated against these two criteria while also considering the impact of changing the optical depth and density of the heated surface.

\subsection{Making a Warm Corona: Testing the Effects of Optical Depth}

The phenomenological fits by Petrucci et al. (2018) found that the warm corona model for the soft excess required that the warm layer have $\tau_{\mathrm{T}} \approx 10-20$. The black solid lines in Figure 1 shows the results of four of our reflection calculations for the case of $n_{\mathrm{H}}=2 \times 10^{14} \mathrm{~cm}^{-3}$ and $\tau_{\mathrm{T}}=10$. The four panels of the figure display the impact of increasing the heating fraction $h_{f}$ from 0.1 to 0.7 . Each panel also illustrates the radiation fields impacting the model slab, with the external cutoff power-law irradiating the surface of the layer shown as the dashed line, and the blackbody injected at the bottom shown as the dotted line. Lastly, to illustrate the impact of the heating injected into the slab, the solid red curve in each panel shows the emission and reflection spectrum of a model with no artificial heating. Every model, including the one with no heating, is subject to the same total energy flux of $D(R)$ ensuring that the only difference between models is how much energy is injected as heat using the $\mathscr{H}$ function (Eq. 1).

Comparing the black and red curves clearly shows the impact of the heat released into the model slab. As the power-law is constant in each model, the spectrum changes as a function of $h_{f}$ simply due to the response of the additional heat injected throughout the layer. This heat leads to two significant effects. First, it results in enhanced Compton up-scattering of the blackbody throughout the layer, and, second, it increases the overall ionization state of metals in the gas. Even at the low level of $h_{f}=0.10$, Compton up-scattering of the blackbody creates an excess of emission between 0.1 and $\approx 0.7 \mathrm{keV}$, although the temperature of the slab $(k T \lesssim 0.2 \mathrm{keV})$ is low enough that significant recombination line emission is imprinted on the spectrum, and the hard X-ray spectrum is nearly unaltered. At this small level, the gas and resulting spectral properties are still dominated by the effects of the powerlaw.

The impact of the heating is seen more clearly in the $h_{f}=0.3$ model, where a stronger soft excess is now apparent, along with reduced line emission below $\sim 1 \mathrm{keV}$. Crucially, the $\mathrm{Fe} \mathrm{K} \alpha$ line is now significantly stronger than in the model with no heating. In both cases it originates from recombination onto He-like Fe, but the extra heat in the $h_{f}=0.3$ model has increased the population of highly ionized iron in the gas, leading to a much stronger line.

Both of these effects are further enhanced in the $h_{f}=0.50$ model, where the reflection spectrum now clearly exhibits a strong, and relatively featureless, soft excess below $\sim 2 \mathrm{keV}$. To better understand the physics leading to this spectrum, Figure 2 plots the heating and cooling rates for all the processes considered by the model as a function of $\tau_{\mathrm{T}}$. The solid horizontal line at the top of the plot shows that $\mathscr{H}$ (Eq. 1), the coronal heating injected into the layer, dominates the heating of the gas at all depths, even close to the surface. Cooling of the gas is dominated by Compton scattering for $\tau_{\mathrm{T}} \gtrsim 3$, and by bremsstrahlung at lower Thomson depths. Indeed, as seen below, free-free cooling, which increases as $T^{1 / 2}$ (e.g., Rybicki \& Lightman 1979), becomes increasingly important in shaping the spectrum as $h_{f}$ increases. Fig. 2 also shows the temperature of the slab (solid black line and right-hand axis), which varies from $\approx 0.2 \mathrm{keV}$ at the bottom to $\approx 0.85 \mathrm{keV}$ at the surface. Therefore, the temperature of this $\tau_{\mathrm{T}}=10$ slab is consistent with the properties of a warm corona inferred by spectral modeling (e.g., Petrucci et al. 2018), and also appears to produce a strong and relatively featureless soft excess.

The reflection and emission spectrum of the slab changes dramatically in the $h_{f}=0.70$ model. In this case, the gas is heated so much by the coronal heating that bremsstrahlung and Compton cooling dominate the other cooling processes by approximately an order of magnitude, and the emission spectrum is dominated by a Comptonized bremsstrahlung continuum with an $\mathrm{Fe} \mathrm{K} \alpha$ line superimposed on the rapidly declining tail of the spectrum. The external hard X-ray power-law only influences the spectrum at energies $\gtrsim 10 \mathrm{keV}$. The temperature of the slab varies from $0.4 \mathrm{keV}$ to $1.4 \mathrm{keV}$, so it is not particularly different from the $h_{f}=0.5$ model. However, the cooling processes in the gas have sufficient temperature sensitivity that relatively modest increases in the gas temperature will significantly alter the emission spectrum. Clearly, there is a maximum amount of coronal heating that can produce a warm corona with the correct properties and yield an appropriate spectral shape.

The effects of doubling the optical depth of the model slab to $\tau_{\mathrm{T}}=20$ are shown in Fig. 3. As expected from Eq. 1, doubling $\tau_{\mathrm{T}}$ will reduce $\mathscr{H}$ everywhere by a factor of 2 . Therefore, the model slabs are cooler for each $h_{f}$ as compared to the $\tau_{\mathrm{T}}=10$ model, and a larger $h_{f}$ is needed to produce a strong and smooth soft excess. Fig. 3 shows that only for $h_{f} \gtrsim 0.7$ will a potential soft excess be formed by the thick layer. The gas temperature of the $h_{f}=0.7$ model reaches $\sim 0.5 \mathrm{keV}$ at the surface. Thus, while it is more challenging to form a successful warm corona in the thick $\tau_{\mathrm{T}}=20$ layer, the difficulty in heating the gas also means that it will be very hard to overheat the gas and produce the Comptonized bremsstrahlung spectrum seen in the $h_{f}=0.70, \tau_{\mathrm{T}}=10$ model. Indeed, the $h_{f}=0.8, \tau_{\mathrm{T}}=20$ model produces a spectrum with an acceptable strong and relatively featureless soft excess with a maximum gas temperature of $\sim 0.6 \mathrm{keV}$ (Fig. 4).

\subsection{Making a Warm Corona: Testing the Effects of Density}

Figure 5 shows the results when the density of the $\tau_{\mathrm{T}}=10$ layer has been increased by a factor of 2.5. The increase in density reduces the ionization parameter $\left(\xi=4 \pi F_{\mathrm{X}} / n_{\mathrm{H}}\right.$, where $F_{\mathrm{X}}$ is the flux of the illuminating power-law) of the slab. Thus, the inner few Thomson depths are far less ionized by the X-ray power-law than the lower density case seen in Fig. 1. The impact of the lower ionization states is readily seen in Fig. 5 in the forest of X-ray recombination lines at $\sim 1 \mathrm{keV}$ and the weak neutral $\mathrm{Fe} \mathrm{K} \alpha$ line. The larger density also significantly enhances the cooling rate by 2 -body processes, in particular bremsstrahlung and line cooling. Finally, the coronal heating function $\mathscr{H} \propto n_{\mathrm{H}}^{-1}$ (Eq. 1), so the heating rate provided by a fixed $h_{f} D(R)$ is reduced for a larger density. These three effects all work together so that it is very challenging to heat the $n_{\mathrm{H}}=5 \times$ $10^{14} \mathrm{~cm}^{-3}$ slab close to the temperatures needed to form a warm corona. The highest temperature reached (in the $h_{f}=0.8$ model) is $\approx 0.3 \mathrm{keV}$, and Compton cooling never dominates the cooling rates at any point in the slab. The emission and reflection spectra shown in Fig. 5 show only a modest soft excess forming at the highest $h_{f}$, with several spectral features still remaining. It is apparent that the 


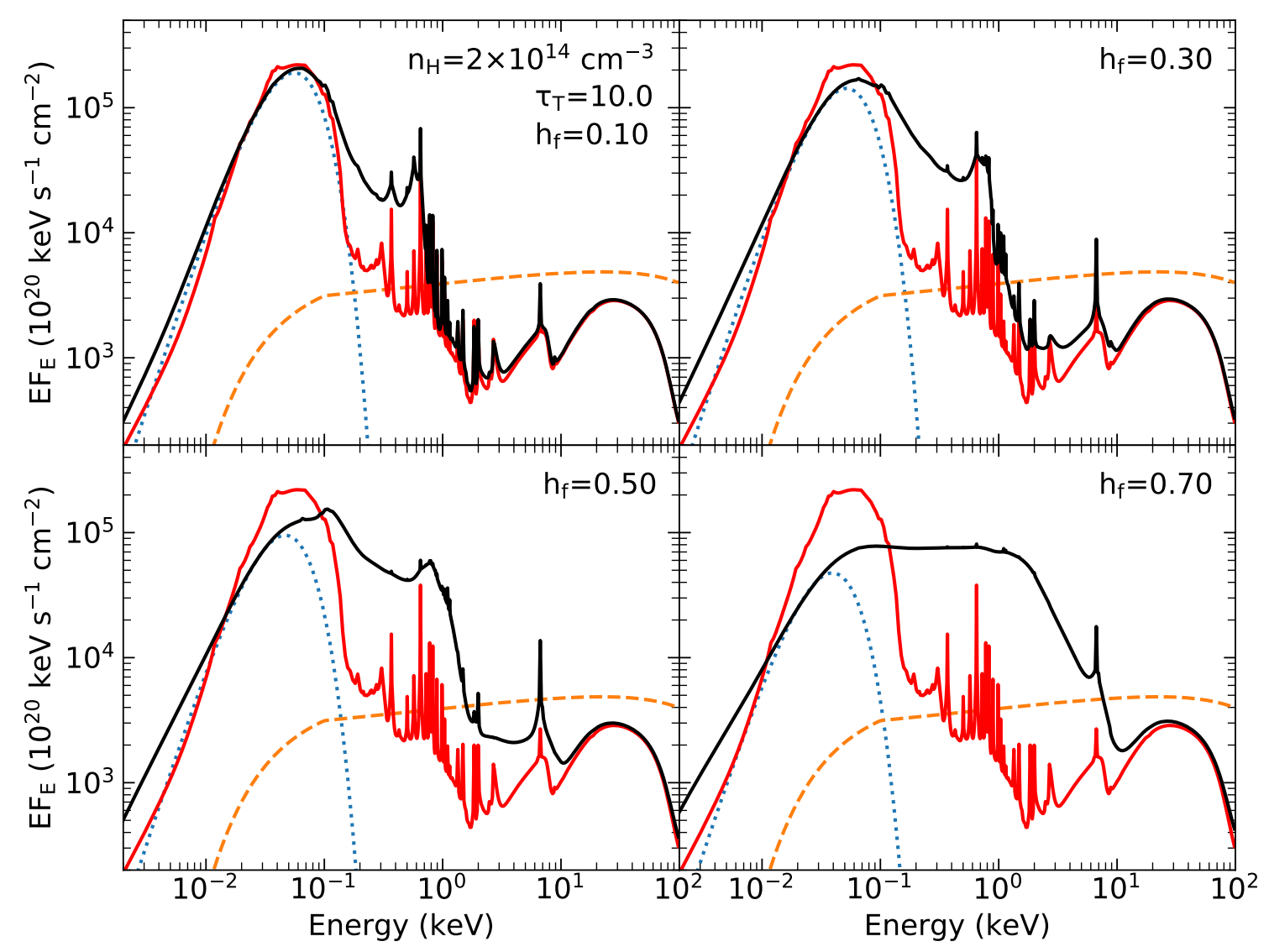

Figure 1. The black curves in each panel show the predicted emission and reflection spectrum from a constant density slab with $n_{\mathrm{H}}=2 \times 10^{14} \mathrm{~cm}^{-3}$ and $\tau_{\mathrm{T}}=10$. The slab is irradiated from above by a fixed cutoff power-law spectrum (dashed curves) and below by a blackbody (dotted curves). The different panels show the effects of increasing the fraction $h_{f}$ of the accretion flux that is injected as heat into the slab (see Sect. 2). The solid red curve in each panel plots the reflection spectrum for the null hypothesis model with no additional heating. In all cases, the power-law, blackbody, and heat fluxes sum to $D(R)$. The figure clearly shows that releasing heat into the surface of the slab strongly affects the shape of the hard X-ray reflection spectrum, including producing a strong soft excess (e.g., when $\left.h_{f}=0.50\right)$.

density at which the coronal heating is injected will be critical in whether or not a warm corona can develop.

\subsubsection{A Hydrostatic Atmosphere}

To further explore the effects of the density on the formation of a warm corona, the calculations were repeated for an irradiated atmosphere in hydrostatic balance ${ }^{1}$. This set of models considers a $\tau_{\mathrm{T}}=10$ layer at the surface of a radiation pressure supported accretion disc 5 Schwarzschild radii away from a $3 \times 10^{7} \mathrm{M}_{\odot}$ black hole accreting at $0.05 \times$ its Eddington rate (Merloni et al. 2000; Ballantyne et al. 2001b). With these parameters $D(R)=$ $3.594 \times 10^{15} \mathrm{erg} \mathrm{cm}^{-2} \mathrm{~s}^{-1}$. As in the constant density models, the illuminating power-law flux is fixed at $0.1 D(R)$ with $\Gamma=1.9$ and

1 Note that this is a different experiment from the one performed by Różańska et al. (2015), as only the heated $\tau_{\mathrm{T}}=10$ layer is in hydrostatic balance. The connection to the cooler accretion disc underneath the layer is neglected.
$E_{\text {cut }}=220 \mathrm{keV}$, and a coronal heating flux $h_{f} D(R)$ is injected into the atmosphere with the heating function $\mathscr{H}$ (Eq. 1).

Figure 6 shows the emission and reflection spectra produced by the heated and irradiated atmospheres. The variation of spectral shape and properties with $h_{f}$ is similar to the constant density case (Fig. 1), with a prominent soft excess forming at $h_{f}=0.5$ and a Comptonized bremsstrahlung continuum seen when $h_{f}=0.7$. However, there are some interesting differences in the spectra due to the density structure adjusting to the heat deposited within it. For example, the overall density of the atmosphere decreases as $h_{f}$ increases. When there is no coronal heating the base density of the atmosphere is $4 \times 10^{15} \mathrm{~cm}^{-3}$, but the extra pressure caused by the heating decreases the density throughout the atmosphere (e.g., the base density is $3 \times 10^{13} \mathrm{~cm}^{-3}$ when $h_{f}=0.5$ ). This large-scale drop in density will reduce the rates of 2-body processes throughout the layer, increasing the temperature and ionization state of the gas, allowing the formation of a warm corona. The lower density also reduces the efficiency of photoelectric absorption, even at low values of $h_{f}$, and thus the reflection spectra of the heated models is 


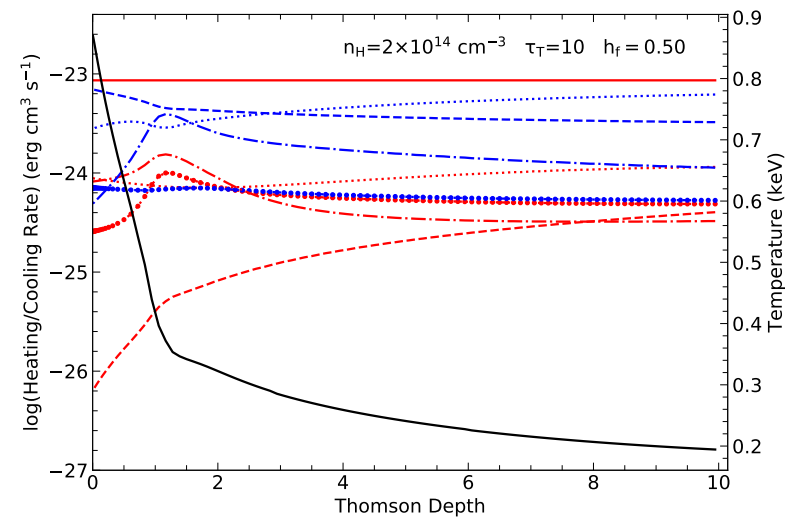

Figure 2. A close look at the heating and cooling processes (red and blue lines, respectively) in the heated slab with $n_{\mathrm{H}}=2 \times 10^{14} \mathrm{~cm}^{-3}, \tau_{\mathrm{T}}=10$, and $h_{f}=0.5$. The different processes are denoted by different line styles: Compton heating and cooling (dotted lines), bremsstrahlung heating and cooling (short-dashed lines), photo-ionization heating and line cooling (dotdashed lines), and recombination heating and cooling (thick dotted lines). The solid horizontal line indicates the heating injected into the layer (Eq. 1) which is constant in this constant density slab. Finally the solid black line and right-hand axis shows the temperature of the slab, indicating that it has an appropriate temperature for a 'warm corona'.

shifted vertically upwards from the non-heated model in the upper two panels of Fig. 6.

The heating/cooling rates and temperature are plotted as a function of $\tau_{\mathrm{T}}$ for the $h_{f}=0.5$ hydrostatic model in Fig. 7. This figure clearly shows the impact of the variable density structure on the properties of the layer. As $\mathscr{H} \propto n_{\mathrm{H}}^{-1}$ (Eq. 1), the heating of the gas increases quickly as the density falls near the surface of the atmosphere. This causes the temperature to rapidly increase to $\sim 1-$ $2 \mathrm{keV}$. This warm, $\tau_{\mathrm{T}} \sim 1$ skin is dominated by Compton cooling and will provide an effective scattering layer for the thermal and line emission emerging from deeper in the atmosphere. These experiments shows that a warm corona could develop in the more realistic density structure of a hydrostatic atmosphere, subject to the same conditions as the constant density slabs (i.e., as long as the density is not too large, and the heating is not too extreme).

\section{DISCUSSION}

\subsection{Conditions Needed to Produce a Warm Corona and a Soft Excess}

The results described above shows that accretion heat dissipated into a $\tau_{\mathrm{T}}=10-20$ gas layer illuminated by an X-ray power-law can, under certain conditions, lead to the temperatures and spectral shapes consistent with a warm corona origin for the AGN soft excess. However, as discussed below, the successful warm corona models exist in a 'Goldilocks' region of parameter space, where the gas can neither be too hot nor too cold. Given the strong density dependence on the heating and cooling rates, these temperature requirements also translate into one on density. The exact values of $n_{\mathrm{H}}$ and $h_{f}$ that lead to a warm corona will also depend on $D(R)$. As a single representative value of $D(R)$ is assumed in this investigation, the discussion on the dependence of the warm corona on $n_{\mathrm{H}}$ and $h_{f}$ will necessarily be qualitative with the understanding that if
$D(R)$ increases over the value used here, the range of $h_{f}$ that leads to the formation of a warm corona will be shifted to smaller values. Similarly, a larger $D(R)$ would allow a warm corona to exist at higher densities than those considered in Sect. 3.2.

Sect. 3.1 and 3.2 showed that specific conditions are needed in order for a warm corona with $k T \sim 1 \mathrm{keV}$ to develop in the heated gas. If the coronal heating was too low than the gas temperature would not rise high enough to fully ionise the metals or for Comptonization to dominate the spectral shape below $\sim 1 \mathrm{keV}$. In these situations, the emitted spectrum would have a modest soft excess overlayed with multiple recombination lines (e.g., top row of Fig. 1). From Eq. 1 it is seen that such weak heating can occur if $h_{f}$ is too small, or if the heated layer is too thick, as in Fig. 3. In the latter situation, where $\tau_{\mathrm{T}}=20$, a plausible warm corona required the majority of the accretion flux $D(R)$ to be released in the layer (e.g., Fig. 4).

On the other hand, Fig. 1 also shows that if $h_{f}$ grows too large, then the heated layer produces a Comptonized bremsstrahlung spectrum that extends into the hard X-ray band. This spectral shape could occur in rare circumstances (see Sect. 4.2), but is unlikely to describe the shape of the soft excess observed in the majority of AGNs. Therefore, if dissipation of accretion energy in the upper layers of the accretion disc is to be considered as a potential origin for the soft excess, its strength must occur within a relatively narrow range so as to provide enough heating to both significantly enhance the rate of Comptonization and ionization, but is limited enough so as to not overheat the gas.

The density of the heated layer also plays a crucial role in the ability of the warm corona to form in the heated layer. As both line-cooling and bremsstrahlung are two body interactions, an increase in density will boost the efficiency of both processes and thus reducing the impact of the heating function $\mathscr{H}$. In addition, as the heat was deposited on a per particle basis (Eq. 1), a fixed heat flux, $h_{f} D(R)$, will have less impact for larger densities. Fig. 5 shows the outcome of the constant density calculations when $n_{\mathrm{H}}=5 \times 10^{14} \mathrm{~cm}^{-3}$, a factor of 2.5 larger than the ones shown in Fig. 1. Even with the largest $h_{f}$ the gas temperature did not exceed $\sim 0.3 \mathrm{keV}$, and a smooth soft excess never appeared in the spectrum. The experiments performed here strongly suggest that a warm corona origin for the soft excess is likely to be only viable in a heated layer that is not very dense. These conditions may be naturally reached if the top $\tau_{\mathrm{T}} \sim 10-20$ layer of an accretion disc falls off in density with height in a manner similar to that needed for hydrostatic balance. Fig. 6 showed that this scenario can produce appropriate soft excesses and warm coronae with $k T \sim 1 \mathrm{keV}$.

The role of the external power-law is also found to be crucial in the formation of a warm corona. The X-ray power-law provides a base level of heat and ionization throughout the gas layer, reducing the atomic opacity at all energies. Thus, the emitted spectra (e.g., Fig. 1), which is the combination of the transmitted blackbody, thermal emission from the gas, and scattered power-law photons, do not exhibit strong absorption lines below $\sim 1 \mathrm{keV}$, as found by García et al. (2019). We verified with our constant density models that the absorption lines appeared upon removing the power-law from the calculation. Thus, if a warm corona exists in AGN accretion discs, it must occur along with the hot corona that produces the hard X-ray power-law. Such a scenario would provide constraints on the vertical transport of accretion energy through the disc.

The predicted emission and reflection spectra show that the impact of a $k T \sim 1 \mathrm{keV}$ warm corona has additional effects on the $\mathrm{X}$-ray spectra beyond producing a smooth soft excess. The extra heating provided by $\mathscr{H}$ increases the ionization state of the gas and 


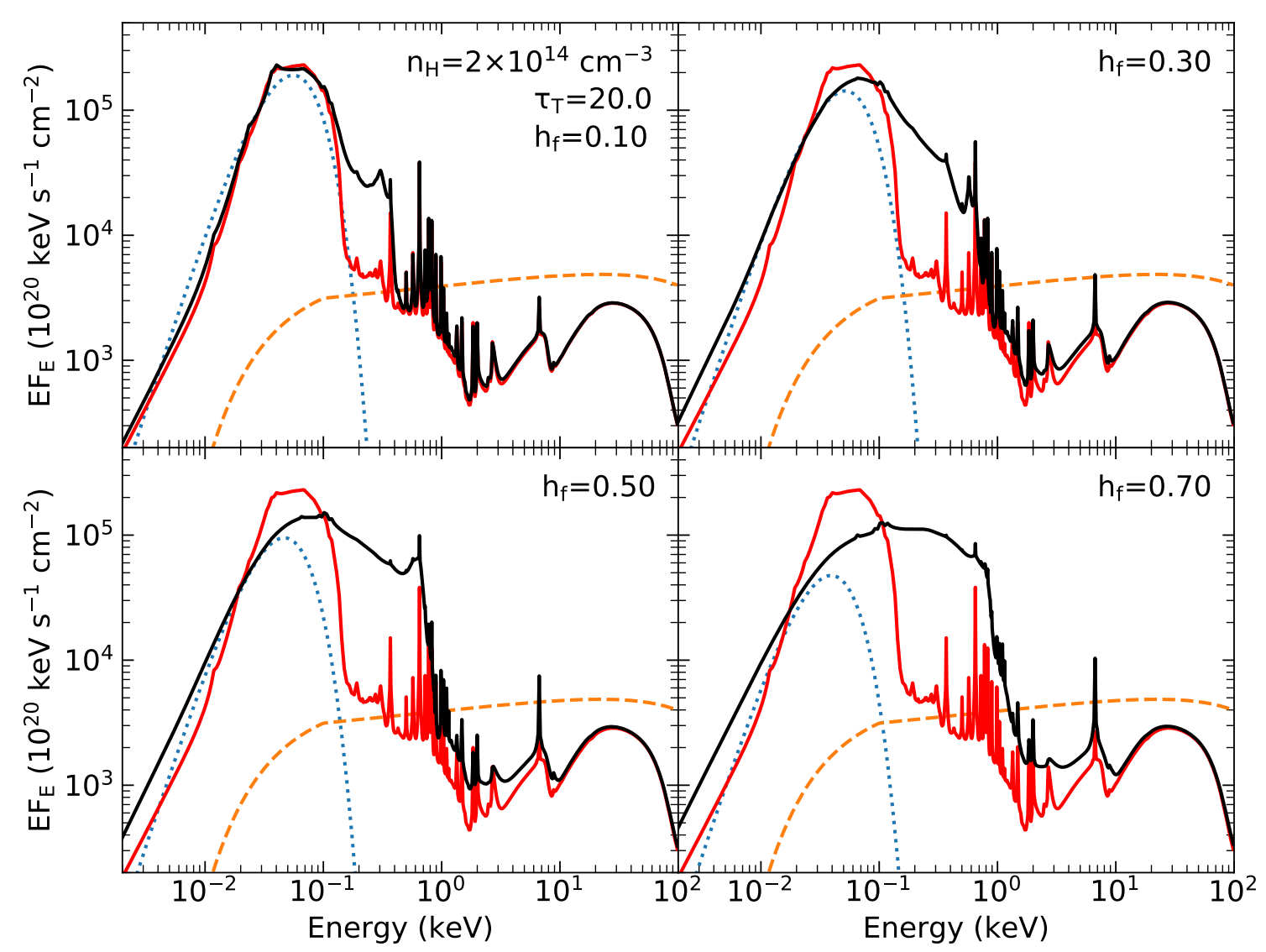

Figure 3. As in Fig. 1, but now for a thicker $\tau_{\mathrm{T}}=20$ layer. As the same heating flux is spread over twice the optical depth, the gas is not heated to the same extent, and it requires a larger $h_{f}$ to obtain a spectrum with a strong soft excess.

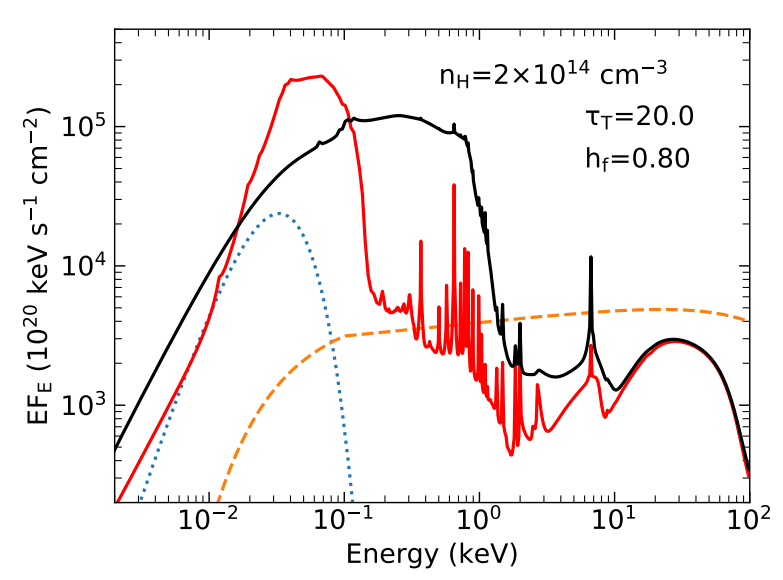

Figure 4. The model results for a $n_{\mathrm{H}}=2 \times 10^{14} \mathrm{~cm}^{-3}$ slab with $\tau_{\mathrm{T}}=20$ and $h_{f}=0.8$. The line styles distinguish the various radiative components as described in the caption to Fig. 1. Very optically thick warm corona can still generate a strong soft excess, but requires that a large fraction of the accretion energy be dissipated in the heated layer. thus an ionised $\mathrm{Fe} \mathrm{K} \alpha$ line at $6.7 \mathrm{keV}$ is prominent in all the output spectra. Ionised $\mathrm{Fe} \mathrm{K} \alpha$ lines are not commonly observed in most Seyfert galaxies, including those with soft excesses (e.g., Walton et al. 2013). This may indicate that a warm corona is not the correct model for producing the AGN soft excess. However, the models calculated here considered only a single radius and one value of $D(R)$, while X-ray observations probe the integrated emission from the inner accretion disc, as well as detecting emission reprocessed from much larger distances (e.g., Patrick et al. 2012). Therefore, a more comprehensive investigation of the warm corona scenario, which accounts for the radial dependence of $D(R)$ and (potentially) $h_{f}$, is needed to better compare to observations. The development of such a model, including the ability to fit AGN X-ray spectra, is planned for future work.

Given that it is possible that both a warm and a hot corona exist in the centers of accretion flows, it appears likely that the soft excess is actually produced by a combination of both Comptonized thermal emission and reprocessed hard X-ray emission. The multiepoch spectral modeling of Mrk 335 by Keek \& Ballantyne (2016) showed that, upon subtracting the emission predicted from reflection, the remaining soft excess flux tracked the overall flux level of the source, indicating that it was influenced by the underlying accretion physics. The soft excesses presented in Sect. 3 are 


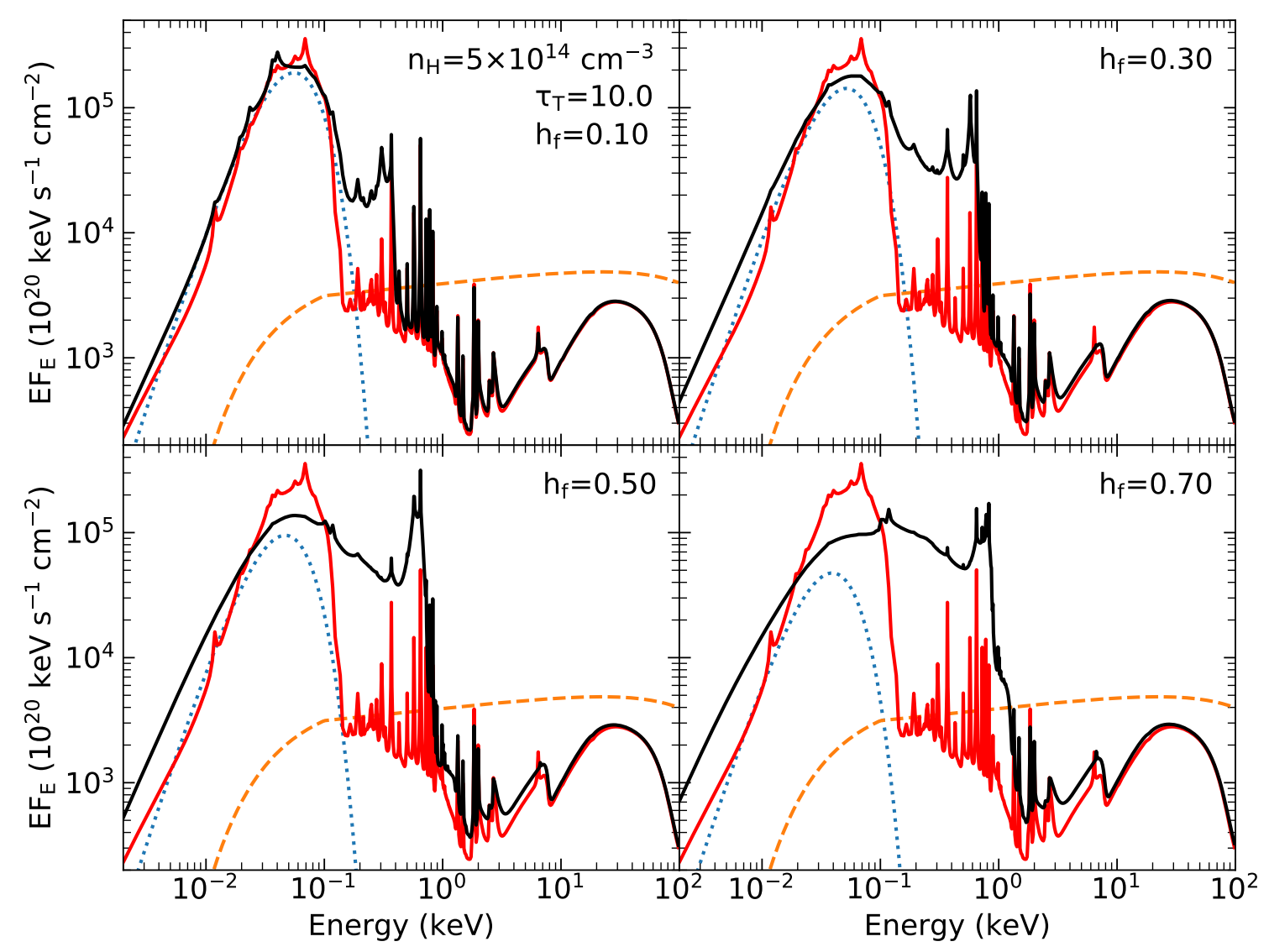

Figure 5. As in Fig. 1, but now for a denser layer with $n_{\mathrm{H}}=5 \times 10^{14} \mathrm{~cm}^{-3}$. The significant increase in density (by $250 \%$ ) reduces the overall ionization and coronal heating rate (Eq. 1) throughout the slab. In addition, cooling via 2-body interactions (e.g., bremsstrahlung) is enhanced, and the slab is able to efficiently cool even at large $h_{f}$. Therefore, the density of the heated layer appears to be crucial in developing a soft excess through a warm corona.

comprised of both emission from the Comptonized blackbody and emission from gas heated by the power-law. We have neglected the influence of relativistic blurring for the purposes of this paper, but if this emission is emerging from close to the inner edge of the accretion disc, then relativistic smearing will further smooth out the predicted soft excesses. This effect will allow even relatively small values of $h_{f}$ to produce smooth soft excesses. The combined effects of both the hard and warm corona, and how they change in strength with radius and accretion rate, may lead to the variety of spectral features and shapes observed in AGNs.

\subsection{A Warm Corona Connection to Narrow-Line Seyfert 1 Galaxies?}

The results of the warm corona calculations showed that the excess heating in a $\tau_{\mathrm{T}}=10-20$ gas layer can produce an interesting range of potential spectral shapes. The most unusual ones were the $h_{f}=0.7$ model shown in Figs. 1 and 6 which are dominated by a Comptonized bremsstrahlung continuum that leads to a steep spectrum at energies $\gtrsim 2 \mathrm{keV}$. It is interesting to consider if such unusual spectra may be related to ones observed in AGNs, in particular Narrow-Line Seyfert 1 galaxies (NLS1s; Gallo 2018).
Figure 8 plots the $h_{f}=0.7$ spectrum from the $n_{\mathrm{H}}=2 \times$ $10^{14} \mathrm{~cm}^{-3}, \tau_{\mathrm{T}}=10$ series of models, but we have now added the illuminating power-law to the reflection spectrum, so that $R$, the reflection fraction, is 0.7 , consistent with typical values observed in AGNs (e.g., Zappacosta et al. 2018). The spectrum shown in the figure has a steep hard X-ray spectrum with a $2-10 \mathrm{keV}$ photon-index of 3.04, an ionised $\mathrm{Fe} \mathrm{K} \alpha$ line and deep iron edge, all properties similar to those observed in several NLS1s (e.g., 1H 0707-495; Ballantyne et al. 2001a; Boller et al. 2002; Zoghbi et al. 2010; Fabian et al. 2012; Liu et al. 2016). Many NLS1s are consistent with being relatively low mass supermassive black holes that are accreting at a high fraction of the Eddington limit (e.g., Williams et al. 2018), suggesting that the steep X-ray spectra are a consequence of cooling of the hot corona due to the intense radiation field produced by the rapidly accreting disc. Alternatively, Fig. 8 suggests that heating of the accretion disc surface and the formation of a warm corona can also explain the interesting features of NLS1s. As the accretion rate increases, the disc is expected to grow thicker, perhaps enveloping some of the hot corona into the surface of the disc. Thus, a large fraction of the overall accretion energy is dissipated within the surface of the disc producing a strong warm corona that would lead to this steep spectrum. In this scenario, less luminous 


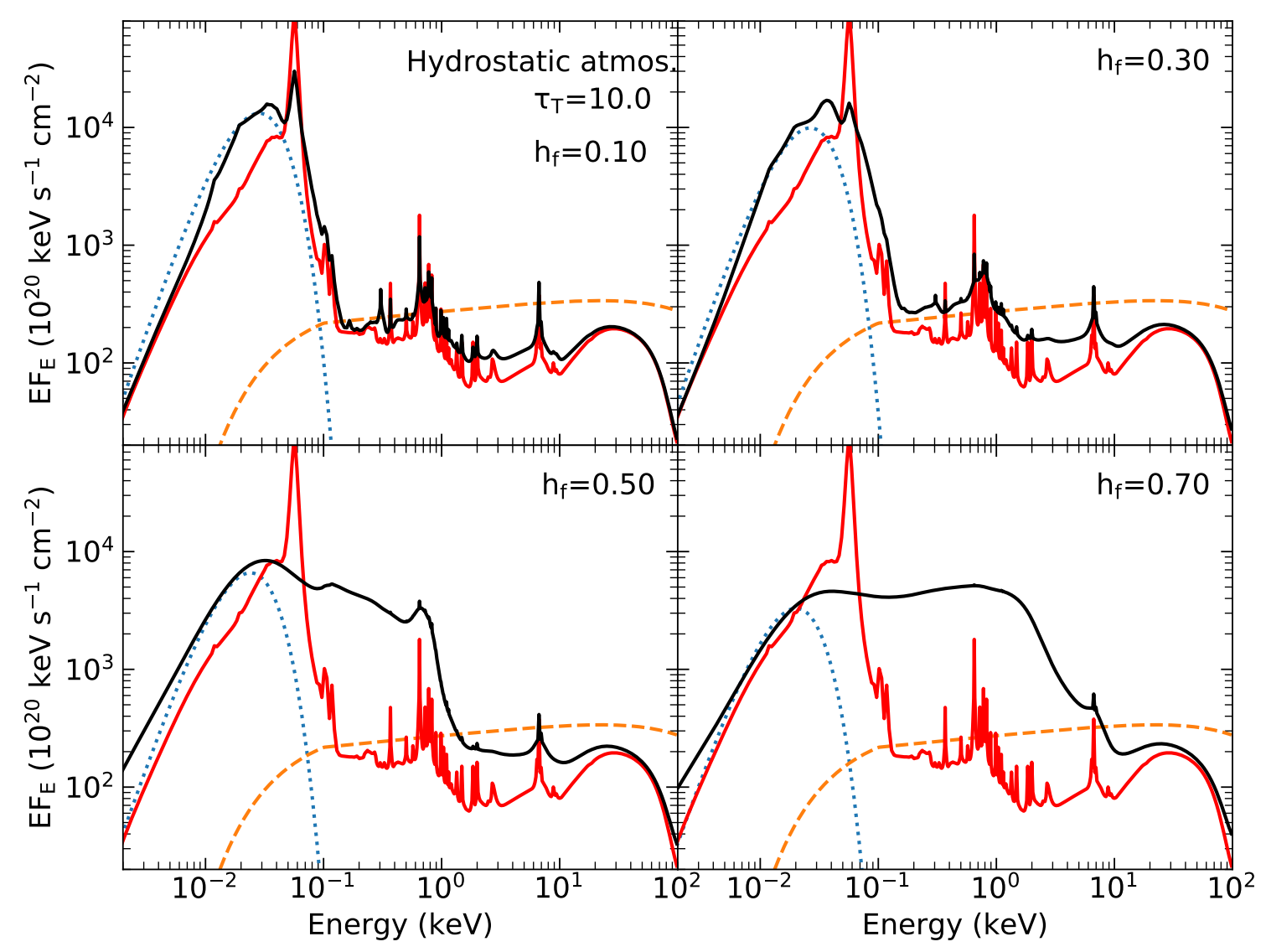

Figure 6. As in Fig. 1, but now the $\tau=10$ layer is forced to be in hydrostatic equilibrium on the surface of a radiation pressure supported accretion disc. The hydrostatic atmosphere is subject to the same radiation conditions and heating processes as the constant density slabs, but with a smaller $D(R)$ (see text). As the atmosphere is in hydrostatic balance, the density falls off significantly with height (Nayakshin et al. 2000; Ballantyne et al. 2001b), reducing the efficiency of the two-body cooling processes, and allowing a strong soft excess to develop when $h_{f} \gtrsim 0.5$.

AGNs, home to less rapidly accreting black holes, would have thinner discs, and therefore smaller $h_{f}$ at their surface yielding moderate warm corona that, along with the reflection spectrum, produce the soft excesses observed in typical AGNs. It appears plausible that if accretion energy is dissipated into the surface layers of the disc, $h_{f}$ would vary as the disc changes in height and density structure, leading to spectral shapes that directly track these effects.

\section{CONCLUSIONS}

The origin of the soft excess observed in the majority of AGN X-ray spectra has remained poorly understood for over three decades. One compelling potential explanation has been that a warm corona is generated at the surface of the accretion disc which Compton scatters thermal emission from the disc into the broad, largely featureless spectrum of the soft excess. A phenomenological model describing this idea can fit the spectra of multiple AGNs and indicates that a temperature of $\sim 1 \mathrm{keV}$ and an optical depth of $\tau_{\mathrm{T}} \approx 10-20$ is needed for a successful warm corona (e.g., Petrucci et al. 2018), raising potential theoretical problems with this scenario (Różańska et al. 2015; García et al. 2019).

This paper considered the physical conditions and emitted spectra of a $\tau_{T}=10$ or 20 gas layer subject to irradiation from a Xray power-law from above, a blackbody from below, and has a variable amount of excess internal heat (proportional to the accretion flux $D(R)$ ). We found that warm corona temperatures and strong, featureless soft excesses could be produced in some models, but only for a particularly narrow range of heating conditions and gas densities. If the internal heating was not sufficient the gas remained too cool and Compton scattering could not produce a strong soft excess; alternatively, if too large a fraction of $D(R)$ is dissipated in the layer, the gas reaches so high a temperature that the emission spectrum is dominated by a Comptonized bremsstrahlung continuum. Similarly, it is found that the density of the gas can not be too large since the gas would be able to efficiently cool through 2-body processes, inhibiting the formation of a warm corona. This density condition may be alleviated if the density of the top $\tau_{T} \approx 10$ layer falls with height as if in hydrostatic balance. Finally, the hard X-ray power-law was found to be crucial in producing a warm corona, as 


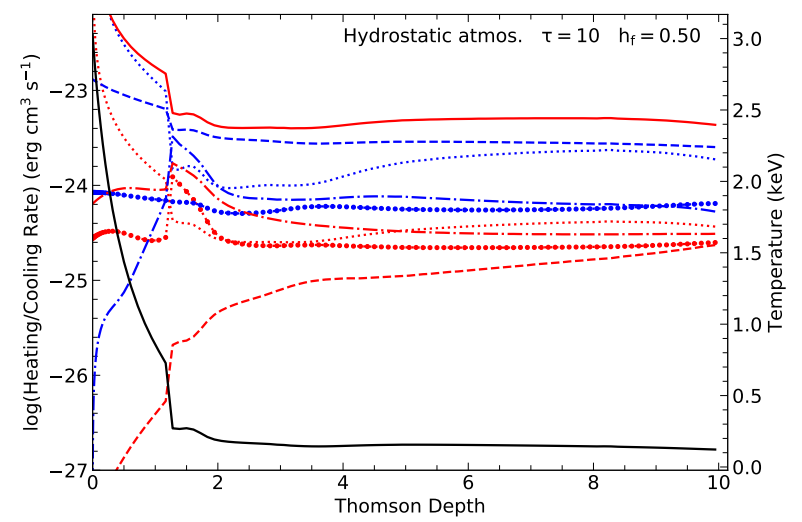

Figure 7. As in Fig. 2, but for a hydrostatic atmosphere with $h_{f}=0.5$. Since the coronal heating function $\mathscr{H} \propto n_{\mathrm{H}}^{-1}$ (Eq. 1), the heating injected into the atmosphere increases strongly at $\tau_{\mathrm{T}} \lesssim 1$ as the density drops with height. This strongly increases the temperature (sold black line), increasing the rate of Compton cooling (dotted blue line), leading to the soft excess in the emission spectrum (Fig. 6).

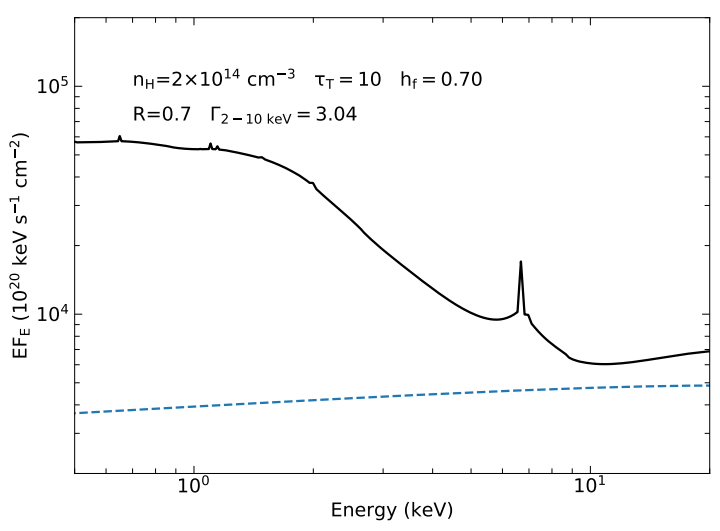

Figure 8. The black line is the emission and reflection spectrum from the $n_{\mathrm{H}}=2 \times 10^{14} \mathrm{~cm}^{-3}, \tau_{\mathrm{T}}=10, h_{f}=0.70$ model upon adding it to the illuminating power-law (dashed line) so that it has a reflection fraction of $R=0.7$. The $2-10 \mathrm{keV}$ photon-index of the resulting spectrum is 3.04 . The warm corona scenario appears to provide a mechanism with which to produce the steep AGN spectra and ionised Fe K $\alpha$ lines seen in NLS1s (e.g., Gallo 2018).

it raised the ionization state of the surface high enough to avoid imprinting the emitted spectrum with strong absorption lines.

Although the conditions for formation are relatively narrow, we conclude that a warm corona is a viable scenario for contributing to the soft excess in AGNs. The spectra calculated here have soft excesses that are produced from the combination of Comptonized disc emission and gas heated by the X-ray power-law. As it is possible for both warm and hot corona to co-exist in AGNs, then the soft excess is likely a product of both origins working together.

Future work on this model will focus on adding the radial dependence of $D(R)$ and generating a model that can be fit to X-ray spectra of AGNs to determine the relative importance of warm and hot corona. In particular, as the properties of the spectra change so dramatically as $h_{f}$ changes, there is the tantalizing possibility of correlating these effects with fundamental AGN parameters such as luminosity and accretion rate. Measuring such changes would lead to important constraints on how accretion energy is transported and dissipated in accretion discs.

\section{ACKNOWLEDGEMENTS}

The author thanks A. Fabian for useful discussions.

\section{REFERENCES}

Arnaud K. A., et al., 1985, MNRAS, 217, 105

Ballantyne D. R., 2004, MNRAS, 351, 57

Ballantyne D. R., 2014, MNRAS, 437, 2845

Ballantyne D. R., Iwasawa K., Fabian A. C., 2001a, MNRAS, 323, 506

Ballantyne D. R., Ross R. R., Fabian A. C., 2001b, MNRAS, 327, 10

Ballantyne D. R., Ross R. R., Fabian A. C., 2002, MNRAS, 336, 867

Bianchi S., Guainazzi M., Matt G., Fonseca Bonilla N., Ponti G., 2009, A\&A, 495, 421

Boller T., et al., 2002, MNRAS, 329, L1

Coppi P. S., 1999, in Poutanen J., Svensson R., eds, Astronomical Society of the Pacific Conference Series Vol. 161, High Energy Processes in Accreting Black Holes. p. 375 (arXiv : astro-ph/9903158)

Crummy J., Fabian A. C., Gallo L., Ross R. R., 2006, MNRAS, 365, 1067

Czerny B., Nikołajuk M., Różańska A., Dumont A. M., Loska Z., Zycki P. T., 2003, A\&A, 412, 317

Fabian A. C., Ross R. R., 2010, Space Sci. Rev., 157, 167

Fabian A. C., et al., 2012, MNRAS, 419, 116

Galeev A. A., Rosner R., Vaiana G. S., 1979, ApJ, 229, 318

Gallo L., 2018, in Revisiting Narrow-Line Seyfert 1 Galaxies and their Place in the Universe. p. 34 (arXiv: 1807.09838)

García J., Kallman T. R., 2010, ApJ, 718, 695

García J. A., Fabian A. C., Kallman T. R., Dauser T., Parker M. L., McClintock J. E., Steiner J. F., Wilms J., 2016, MNRAS, 462, 751

García J. A., et al., 2019, ApJ, 871, 88

Gierliński M., Done C., 2004, MNRAS, 349, L7

Haardt F., Maraschi L., 1991, ApJ, 380, L51

Haardt F., Maraschi L., 1993, ApJ, 413, 507

Jiang Y.-F., Stone J. M., Davis S. W., 2014, ApJ, 784, 169

Jiang J., et al., 2019a, MNRAS, 489, 3436

Jiang Y.-F., Blaes O., Stone J. M., Davis S. W., 2019b, ApJ, 885, 144

Keek L., Ballantyne D. R., 2016, MNRAS, 456, 2722

Kubota A., Done C., 2018, MNRAS, 480, 1247

Leighly K. M., 1999, ApJS, 125, 317

Liu Z., Yuan W., Lu Y., Carrera F. J., Falocco S., Dong X.-B., 2016, MNRAS, 463, 684

Magdziarz P., Blaes O. M., Zdziarski A. A., Johnson W. N., Smith D. A., 1998, MNRAS, 301, 179

Malizia A., Molina M., Bassani L., Stephen J. B., Bazzano A., Ubertini P., Bird A. J., 2014, ApJ, 782, L25

Merloni A., Fabian A. C., Ross R. R., 2000, MNRAS, 313, 193

Molina M., Malizia A., Bassani L., Ursini F., Bazzano A., Ubertini P., 2019, MNRAS, 484, 2735

Nayakshin S., Kazanas D., Kallman T. R., 2000, ApJ, 537, 833

Netzer H., 2019, MNRAS, 488, 5185

Panessa F., et al., 2008, A\&A, 483, 151

Patrick A. R., Reeves J. N., Porquet D., Markowitz A. G., Braito V., Lobban A. P., 2012, MNRAS, 426, 2522

Petrucci P. O., et al., 2013, A\&A, 549, A73

Petrucci P. O., Ursini F., De Rosa A., Bianchi S., Cappi M., Matt G., Dadina M., Malzac J., 2018, A\&A, 611, A59

Pounds K. A., Warwick R. S., Culhane J. L., de Korte P. A. J., 1986, MNRAS, 218, 685

Ricci C., et al., 2017, ApJS, 233, 17

Ricci C., et al., 2018, MNRAS, 480, 1819 
Ross R. R., 1979, ApJ, 233, 334

Ross R. R., Fabian A. C., 1993, MNRAS, 261, 74

Różańska A., Malzac J., Belmont R., Czerny B., Petrucci P. O., 2015, A\&A, 580, A77

Rybicki G. B., Lightman A. P., 1979, Radiative processes in astrophysics

Scott A. E., Stewart G. C., Mateos S., 2012, MNRAS, 423, 2633

Shakura N. I., Sunyaev R. A., 1973, A\&A, 500, 33

Vasudevan R. V., Fabian A. C., 2007, MNRAS, 381, 1235

Walter R., Fink H. H., 1993, A\&A, 274, 105

Walton D. J., Nardini E., Fabian A. C., Gallo L. C., Reis R. C., 2013, MNRAS, 428, 2901

Williams J. K., Gliozzi M., Rudzinsky R. V., 2018, MNRAS, 480, 96

Winter L. M., Veilleux S., McKernan B., Kallman T. R., 2012, ApJ, 745, 107

Zappacosta L., et al., 2018, ApJ, 854, 33

Zoghbi A., Fabian A. C., Uttley P., Miniutti G., Gallo L. C., Reynolds C. S., Miller J. M., Ponti G., 2010, MNRAS, 401, 2419

This paper has been typeset from a $\mathrm{T}_{\mathrm{E}} \mathrm{X} / \mathrm{L} \mathrm{T}_{\mathrm{E}} \mathrm{X}$ file prepared by the author. 